\title{
Diagnostic and prognostic value of circulating tumor DNA in gastric cancer: a meta-analysis
}

\author{
Yunhe Gao ${ }^{1, *}$, Kecheng Zhang ${ }^{1, *}$, Hongqing $\mathrm{Xi}^{1, *}$, Aizhen $\mathrm{Cai}^{1}$, Xiaosong $\mathrm{Wu}^{1}$, Jianxin \\ Cui ${ }^{1}$, Jiyang $\mathrm{Li}^{1}$, Zhi Qiao', Bo Wei ${ }^{1}$, Lin Chen ${ }^{1}$ \\ ${ }^{1}$ Chinese PLA General Hospital, Department of General Surgery, Beijing, 100853, People's Republic of China \\ *These authors contributed equally to this work \\ Correspondence to: Lin Chen, email: chenlinbj@sina.com
}

Keywords: ctDNA, gastric cancer, diagnosis, prognosis, meta-analysis

Received: September 26, $2016 \quad$ Accepted: December 13, 2016

Published: December 21, 2016

\begin{abstract}
Background: Circulating tumor DNA (ctDNA) has offered a minimally invasive approach for detection and measurement of gastric cancer (GC). However, its diagnostic and prognostic value in gastric cancer still remains unclear.

Results: A total of 16 studies comprising 1193 GC patients met our inclusion criteria. The pooled sensitivity and specificity were 0.62 ( $95 \%$ confidence intervals (CI) 0.59-0.65) and 0.95 (95\% CI 0.93-0.96), respectively. The AUSROC (area under SROC) curve was 0.94 (95\% CI 0.89-0.98). The results showed that the presence of certain ctDNA markers was associated with larger tumor size (OR: 0.26, 95\% CI 0.11-0.61, $p=0.002)$, TNM stage (I + II/III + IV, OR: 0.11, 95\% CI 0.07-0.17, p = 0.000), as well as $H$. pylori infection. (H.p negative/H.p positive, OR: $0.57,95 \%$ CI $0.36-0.91$, $p=0.018$ ). Moreover, there was also a significant association between the presence of ctDNA and worse overall survival (HR 1.77, 95\% CI 1.38-2.28, $p<0.001$ ), as well as disease-free survival (HR 4.36, 95\% CI 3.08-6.16, $p<0.001$ ).

Materials and Methods: Pubmed, Embase, Cochrane Library and Web of Science databases were searched for relating literature published up until November 30, 2016. Diagnostic accuracy variables were pooled by the Meta-Disc software. Engauge Digitizer and Stata software were applied for prognostic data extraction and analysis.

Conclusions: Our meta-analysis indicates the detection of certain ctDNA targets is significantly associated with poor prognosis of GC patients, with high specificity and relatively moderate sensitivity.
\end{abstract}

\section{INTRODUCTION}

Gastric cancer (GC) remains the fourth most common cancer and the second leading cause of cancer-related death in the world [1]. Although recent achievements in cancer diagnosis and therapy strategies have improved the clinical outcomes, a total of 950,000 new GC cases and 720,000 deaths related to GC were estimated to occur in 2014 worldwide [2]. Many patients are diagnosed with $\mathrm{GC}$ in its advanced stage due to the lack of early diagnostic techniques. Failure to identify patients with high-risk of metastasis and recurrence has also resulted in an unsatisfactory prognosis of GC patients.

Tumorigenesis and cancer progression involves a series of biological processes. During these complex events, cell-free DNA (cfDNA) might be released into the bloodstream by cells undergoing apoptosis or necrosis, as well as by exosomes [3]. The cfDNA derived from tumors, also known as circulating tumor DNA (ctDNA), contains different fragments of tumor gene, which reflect specific genetic alterations of cancer, such as methylation or mutation. These molecular alterations are analyzed by various strategies, including polymerase chain reaction followed by sequencing analyses or by methylationspecific PCR and digital PCR [4, 5]. Other researchers have also demonstrated the value of specific ctDNA in diagnosis or as a prognosis indicator for cancer. In nonsmall cell lung cancer (NSCLC), colon cancer, breast cancer, and other malignancies, the presence of certain ctDNA markers was found to be an indicator and predictor of tumor progression or drug resistance [6,7]. Although many recent studies have focused on the relationship between cfDNA/ctDNA and GC, the results are still unclear. Therefore, this study initiated a comprehensive 
analysis to clarify the precise value of ctDNA in GC patient diagnosis and prognosis.

\section{RESULTS}

\section{Study selection process}

As shown in Figure 1, 16 studies were eligible for system review after carefully screening and re-checking by the entire research group. The details and main characteristics of included studies are summarized in Table 1. The 16 eligible studies contained a total of 1193 patients with a median sample size of 69 (range: 40-202, mean: 73) and were published between 2005 and 2016. Among them, 13 studies enrolled patients from East Asian countries/areas (one from Hong Kong, one from Thailand, two from Japan and the remaining 10 from People's Republic of China). Two studies were performed in Greece and one was in Iran. The two studies from the same medical center in Greece (University General Hospital of Alexandropolis) involved different gene targets (SOX17, APC and RASSF1A); therefore, we considered them as independent studies. The genes in the analysis and their main function were classified according to Simone M's research [24] (Figure 2) and other detail information is showed in Supplementary Table S1. The results of diagnosis quality assessment are showed in Figure 3 and the detail information was displayed in Supplementary Table S2. The results of quality assessment according to NOS scale were presented in Supplementary Table S3 and all studies have achieved a score over 5 stars.

\section{Detection of ctDNA}

Several molecular detection methods, such as methylation-specific PCR (MSP) or quantitative MSP (qMSP), were applied in enrolled studies. One study [15] used bisulfite genomic sequence and another [23] used Taqman PCR to measure the status of specific circulating DNA in GC patients. The blood volume used for DNA detection varied between $400 \mathrm{ul}-5 \mathrm{ml}$ with a median volume of $1.5 \mathrm{ml}$. All the studies collected blood samples before surgery except Henji's study, which withdrew patients' blood during surgery. Fourteen out of all studies extracted DNA from patient serum and the other two from plasma. All the studies focused on circulating gene methylation/hypermethylation.

\section{Meta-analysis results of diagnostic value}

Fifteen studies were pooled for the meta-analysis of diagnostic accuracy. As presented in Figure 4, the overall sensitivity and specificity was 0.62 (95\% CI $0.59-0.65)$ and 0.95 (95\% CI $0.93-0.96)$, respectively. The pooled PLR and NLR were 12.93 (95\% CI 6.28-26.64) and 0.37 (95\% CI 0.26-0.52), respectively. The area under the SROC was 0.94 (95\% CI 0.89-0.98) and the DOR was 28.7 (95\%CI 15.09-54.67). Significant heterogeneity was observed in the diagnostic analysis of 15 studies (sensitivity: $I^{2}=95.3 \%, p=0.000$; specificity: $I^{2}=84.7 \%$, $p=0.000$ ). Analysis of diagnostic threshold showed no significant threshold effect existed with the Spearman correlation coefficient of 0.39 and $p$ value of 0.122 . Therefore, subgroup analysis (Table 2A) was conducted according to different parameters: sample size $(\geq 65$ versus $<65$ ), sample source (plasma versus serum), race (Caucasian versus Asian), and gene targets (single versus combined). However, only a lower heterogeneity was detected in the subgroup of Caucasian race (specificity: $I^{2}$ $=0 \%, p=1.0$ ) and single gene target (specificity: $I^{2}=0 \%$, $p=0.867$ ). In the subgroup analysis for DOR, similar trend was also found in the qPCR group and Caucasian race (qPCR: $I^{2}=0 \%, p=0.413$; Caucasian race: $I^{2}=0 \%$, $p=0.870)$. These results suggested that ctDNA detection method, race and gene target's combination type might be part of the heterogeneity source. The other measures of diagnosis value for subgroup analysis are summarized in A. Meta-regression analysis based on those four factors were also applied to explore the heterogeneity source. However, none of those factors would significantly alter the heterogeneity of universal diagnostic value. (Table 2B) Taken together, we considered race, detection method and gene targets combination as part of the heterogeneity source. And more well-design experiments with consistent methodology in different races are needed to clarify ctDNA's diagnostic role in GC patients.

\section{Meta-analysis results of prognostic significance}

A total of 10 studies were pooled for meta-analysis of survival. Among them, 6 studies were available for calculating overall survival and 4 for disease-free survival. The results showed that high level of ctDNA in GC was associated with worse overall survival (HR: 1.77, 95\%CI 1.38-2.28, $p<0.001)$. A moderate but insignificant heterogeneity was observed $\left(I^{2}=42.0 \%, p=0.111\right)$, so a fixed-effect model was applied during calculation (Figure 6A).

Similarly, a significant association was found between ctDNA presence and GC patients' disease-free survival (HR: 4.69, 95\%CI 2.23-9.85, $p<0.001$ ) with a detectable heterogeneity (Figure 6B, $I^{2}=70.6 \%, p=0.017$ ). Galbraith plot was performed to explore the source of heterogeneity, and the results showed that Yu JL's study might cause the study heterogeneity (Figure 7A). After removing Yu JL's study, heterogeneity for DFS analysis decreased to an insignificant level $\left(I^{2}=48.8 \%, p=0.142\right)$, while the association between ctDNA and DFS remain significant (Figure 7B, HR:4.09, 95\% CI 2.89-5.81, $p<0.001$ ).

\section{Association of ctDNA detection with clinicopathological characteristics of GC patients}

The association between ctDNA detection and major clinicopathological features was assessed using 12 studies. As shown in Table 3, ctDNA presence was significantly 
associated with TNM stage (I+II/III+IV, OR: $0.11,95 \%$ CI $0.07-0.17, p=0.000)$. In detail, ctDNA presence had a significant association with tumor depth (I + II/III + IV, OR: $0.18,95 \%$ CI $0.07-0.45, p=0.001$ ), more lymph node metastasis (N0/N1-3, OR: $0.19,95 \%$ CI $0.06-0.64$, $p=0.008$ ), as well as distant metastasis (M0/M1, OR: 0.32 , $95 \%$ CI $0.20-0.53, p<0.001)$. A significant association was also deteced between $H$. pylori infection and ctDNA presence. (H.p negative/H.p positive, OR: 0.57, $95 \%$ CI $0.36-0.91, p=0.018)$. GC patients with larger tumor load were more likely to have detectable ctDNA (tumor size $<5 \mathrm{~cm} />5 \mathrm{~cm}$, OR: $0.26,95$ CI $0.11-0.61$, $p=0.002)$. Meanwhile, no statistical association was observed between ctDNA and sex (male/female, OR: $1.11,95 \%$ CI $0.84-1.46, p=0.476$ ), Lauren classification (intestinal/diffuse, OR: $0.89,95 \%$ CI $0.57-1.4, p=0.628$ )

\section{Publication bias}

The publication bias was assessed in the association of ctDNA and OS in GC patients (Figure 8). Egger's tests and Begg's tests showed that publication bias was not significant for the enrolled studies (Egger's test: $p=0.233$; Begg's test: $p=0.176$ ).

\section{DISCUSSION}

Traditional surgical/biopsy specimens are used in cancer diagnosis and considered as the gold standard for clinical examination. Pathology results according to surgical/biopsy specimens would also provide fundamental information for clinical decision-making. However, the limitations of specimen source directly from tumor, invasive procedure and delayed reflection for tumor dynamic change have restricted its appliance [25]. Therefore, liquid biopsy has been recently extensively investigated as one of the new diagnostic techniques [26].

Since the first report of fragmented DNA in the whole blood by Mandel and Metais [27] in 1948, cfDNA and ctDNA have been applied in a variety of disciplines. For example, detection of epidermal growth factor receptor (EGFR) T790M mutation in plasma is an effective method to determine EGFR status in NSCLC [5], providing a more expedient measure to predict resistance to EGFR tyrosine kinase inhibitors and prognosis [28]. However, the relationship between ctDNA and GC still remains unclear. Therefore, it is necessary to conduct comprehensive analysis illuminating the clinical utility of ctDNA in GC patient diagnosis and prognosis prediction.

In terms of test sensitivity, the present evidence showed no superiority of ctDNA over conventional protein biomarkers, such as CEA, CA125 and CA724, the combination of which had a diagnosis sensitivity of nearly $60-75 \%$ [29].Therefore, more accurate circulating gene targets need to be defined. On the other hand, the present ctDNA is considered to be more specific for certain types of cancer compared with normal tissue, mainly because the somatic cancer mutations have been identified by their presence in tumor DNA and absence in matched normal DNA [3]. Our analysis confirmed that detection of ctDNA had an

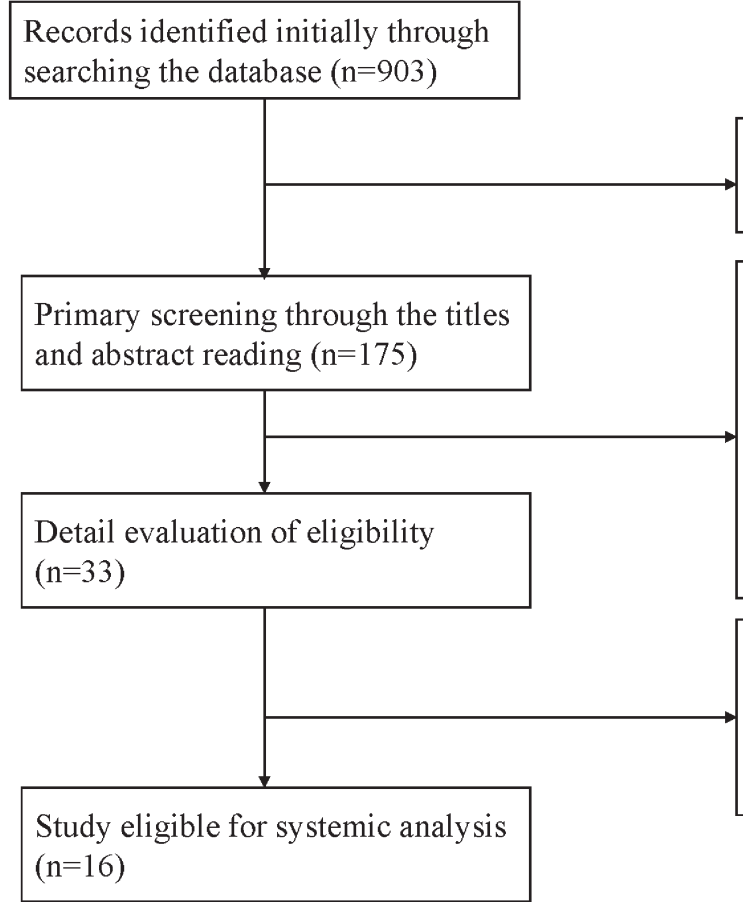

728 Publications irrelevant to circulating DNA or gastric cancer were excluded

142 publications were excluded:

36 circulating cells articles

34 other type of cancer

30 not gene alteration in blood

14 benign diseases

15 reviews

10 not English writing

3 repeated reports

17 studies were excluded

10 studies didn't provide relevant data

6 studies was fundamental experiments

1 study focused on gene mutation in blood

Figure 1: Flow chart of selection process to enroll eligible studies. 
Table 1: Major characteristics of enrolled studies

\begin{tabular}{|c|c|c|c|c|c|c|c|c|c|c|c|c|}
\hline No. & Study & Number & $\begin{array}{l}\text { Sex } \\
(M / F)\end{array}$ & region & $\begin{array}{l}\text { Detection } \\
\text { method }\end{array}$ & Target gene & HR & Follow up & AT & SS & ST & BV (ml) \\
\hline 1 & WK Leung $^{8}$ & 60 & / & Hongkong & MSP & APC/E-cadherin & OS:3.38(1.42-8.05) (F) & $8(0-40)$ & Methylation & Serum & BS & 0.8 \\
\hline 2 & Mohammad R.A. ${ }^{9}$ & 52 & $38 / 14$ & Iran & MSP & P16 & l & I & Hypermethylation & Serum & BS & l \\
\hline 3 & Wang $\mathrm{YC}^{10}$ & 47 & $29 / 18$ & China & MSP & RASSF1A & l & I & Hypermethylation & serum & BS & 5 \\
\hline 4 & Chouhei.S ${ }^{11}$ & 65 & $37 / 28$ & Japan & qMSP & RUNX3 & l & I & Methylation & Serum & BS & / \\
\hline 5 & Kenji H. ${ }^{12}$ & 73 & $57 / 16$ & Japan & qMSP & TFPI2 & l & I & Methylation & Serum & TOS & l \\
\hline 6 & Ioanna B. ${ }^{13}$ & 73 & $51 / 22$ & Greece & MSP & SOX17 & OS:1.60 (1.0-2.55) (F) & $56(20-111)$ & Methylation & Serum & BS & I \\
\hline 7 & Yang $\mathrm{QF}^{14}$ & 40 & $33 / 7$ & China & BGS & BCL6B & OS:1.86 (0.68-5.10) (K) & l & Hypermethylation & Plasma & BS & 1 \\
\hline 8 & Zhi QL ${ }^{15}$ & 202 & $120 / 82$ & China & MSP & XAF1 & DFS:5.71 (3.474-9.383) (K) & l & Methylation & Serum & BS & l \\
\hline 9 & Han $\mathrm{J}^{16}$ & 92 & $53 / 39$ & China & qMSP & MINT2 & DFS:3.362 (1.779-5.981) (D) & / & Methylation & Serum & BS & / \\
\hline 10 & Wu YC & 92 & $53 / 39$ & China & qMSP & P16 & DFS: $2.31(1.00-5.37)(\mathrm{K})$ & I & Methylation & Serum & BS & l \\
\hline 11 & Yu JL ${ }^{17}$ & 92 & $54 / 38$ & China & MSP & TIMP-3 & $\begin{array}{l}\text { DFS:97.376 (8.388- } \\
1130.378) \text { (D) }\end{array}$ & / & Methylation & Serum & BS & l \\
\hline 12 & Zhang $\mathrm{H}^{18}$ & 41 & $30 / 11$ & China & MSP & Spastic paraplegia-20 & l & I & Hypermethylation & & BS & 2 \\
\hline 13 & Chang $\mathrm{L}^{19}$ & 42 & $30 / 12$ & China & MSP & SFRP1 & l & I & Methylation & Serum & BS & 5 \\
\hline 14 & Ioanna B. $(\mathrm{APC})^{20}$ & 73 & $51 / 22$ & Greece & MSP & $\mathrm{APC}$ & OS: $2.94(1.33-6.53)(\mathrm{F})$ & $56(12-111)$ & Methylation & Serum & BS & l \\
\hline \multirow{3}{*}{15} & Ioanna B.(RASSF1A) ${ }^{20}$ & 73 & $51 / 22$ & Greece & MSP & RASSF1A & $\begin{array}{l}\text { OS: } \\
0.87(0.46-1.66)(\mathrm{F})\end{array}$ & $56(12-111)$ & Methylation & Serum & BS & / \\
\hline & Charinya P (PCDH10) $)^{21}$ & 101 & $44 / 57$ & Tailand & MSP & PCDH10 & OS:3.47(1.69-7.11) (F) & l & Methylation & plasma & BS & l \\
\hline & Charinya P (RASSF1A) ${ }^{21}$ & 101 & $44 / 57$ & Thailand & MSP & RASSF1A & OS:1.66(0.98-2.83) (F) & l & Methylation & plasma & BS & l \\
\hline 16 & $\mathrm{Li} \mathrm{WH}^{22}$ & $48(25)$ & $39 / 9$ & China & MSP & OSR2:VAV3:PPFIA3 & / & / & Methylation & Serum & BS & 0.4 \\
\hline
\end{tabular}

K: extracted and calculated from the Kaplan-Meier curves in the studies; F: calculated by the formula provided by Parmar et al ${ }^{37}$. D: directly extracted by the authors in the studies; HR: hazard ratio; AT: alteration type; SS: sample source; ST: sample time; BV: blood volume; MSP: methylation-specific PCR; qMSP: quantitative methylation-specific PCR; BS: before surgery; TOS: time of surgery; OS: overall survival; DFS: disease-free survival.

obvious advantage in GC diagnosis specificity (specificity: 0.95, 95\% CI 0.93-0.96). According to the suggested guidelines for the interpretation of the AUSROC value [30], ctDNA presence in patients had a relatively high diagnostic ability (AUC > 0.9) to indicate the risk of GC (Figure 5).

With regards to prognostic value, the detection of ctDNA in GC was significantly associated with both disease-free survival and overall survival. This finding will strongly strengthen the value of ctDNA in clinical management of patients with GC. The result of DFS part needs to be interpreted with caution due to limited enrolled study number. Moreover, detectable ctDNA was also associated with tumor size and TNM stage, which could be explained by the theory that circulating tumor genes are associated with tumor burden and invasion in patients [3].

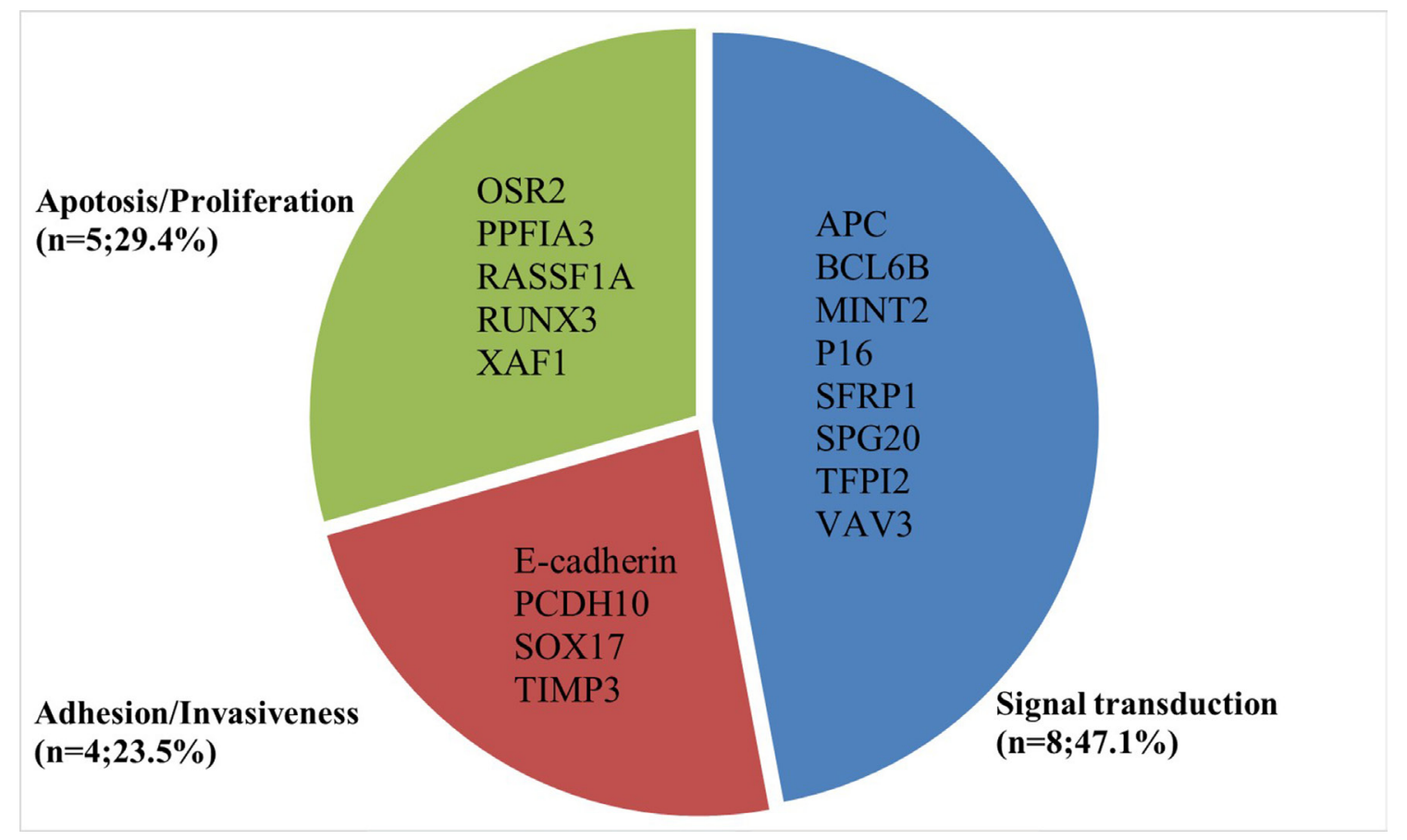

Figure 2: Summarized genetic alterations arranged by main gene function. 
Proportion of studies with low, high or unclear risk of bias

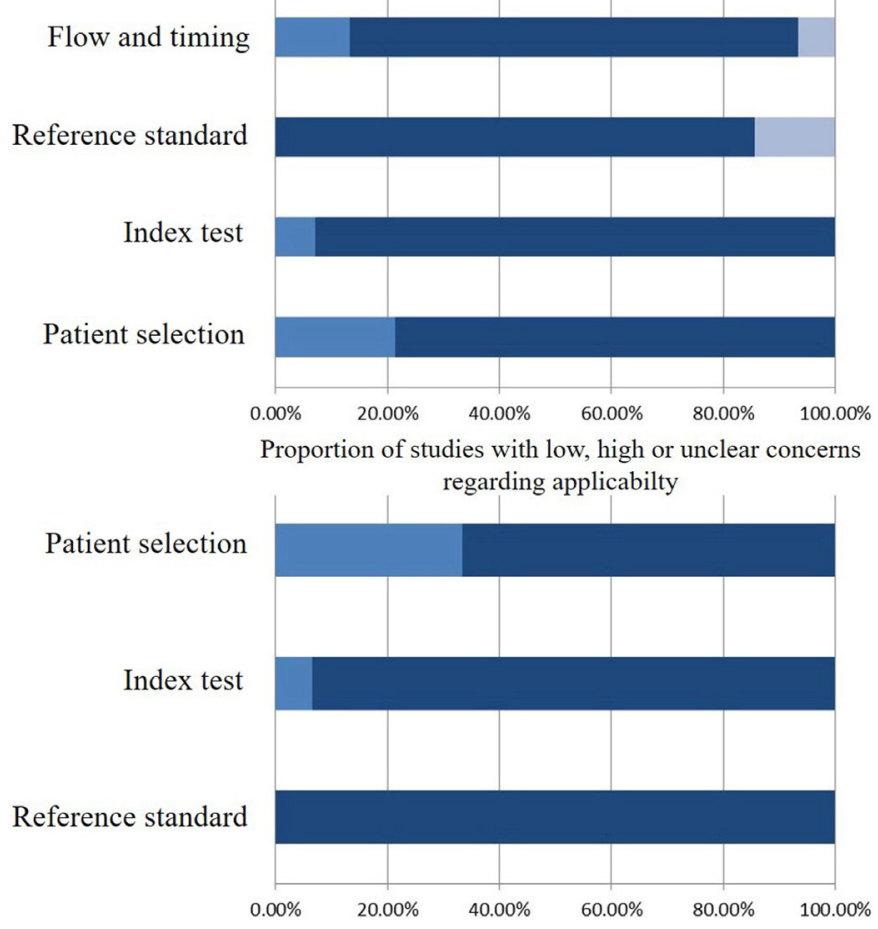

Figure 3: Diagnosis quality assessments of included studies using the QUADAS-2 tool criteria.

A

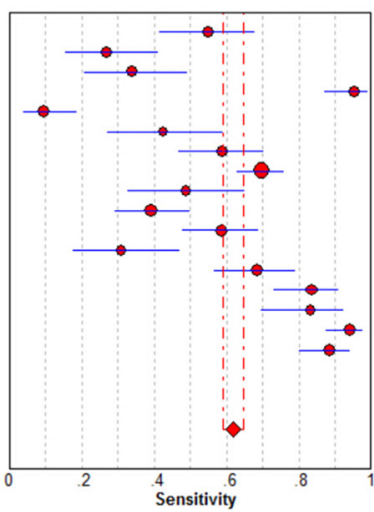

$\mathrm{C}$

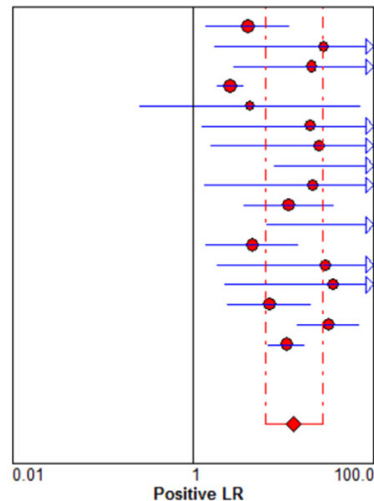

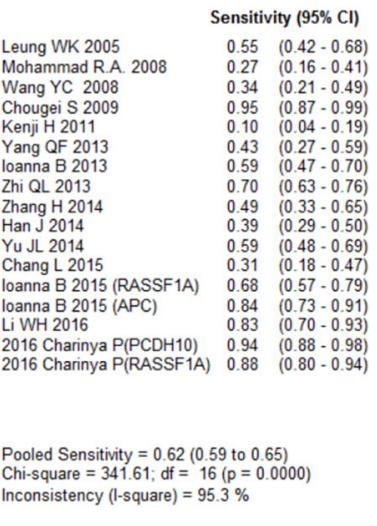

Positive LR (95\% CI)

Leung WK 2005 Mohammad R.A. 2008 Wang YC 2008 Chougei S 200 Yang OF 2013 Yang QF 2013 Zhi QL 2013 Zhang $\mathrm{H} 2014$ Han J 2014 Yu JL 2014 Chang $L 2015$ loanna B 2015 (RASSF1A) loanna B 2015 (APC) Li WH 201 2016 Charinya $\mathrm{P}(\mathrm{PCDH} 10)$
2016 Charinya P(RASSF1 $\begin{array}{lc}4.03 & (1.37-11.83) \\ 27.91 & (1.71-455.64)\end{array}$ $\begin{array}{ll}27.91 & (1.71-455.64) \\ 20.43 & (2.81-148.51)\end{array}$ $2.60(1.86-3.64)$ $\begin{array}{ll}2.60 & (1.86-3.64) \\ 4.26 & (0.25-71.52)\end{array}$ $19.63(1.24-311.58)$ $24.69(1.59-384.38)$ $124.07 \quad(7.81-1,971.17)$ $21.48 \quad(1.36-338.50)$ $11.48 \quad(3.67-35.92)$ $104.31(6.54-1.663 .50)$ $4.54(1.39-14.80)$ $\begin{array}{ll}28.66 & (1.85-445.24) \\ 34.91 & (2.25-540.87)\end{array}$ $34.91(12.25-540.87)$ $6.94(2.38-20.23)$
$31.67 \quad(14.38-69.75)$ $10.82 \quad(6.82-17.16)$

Random Effects Model Pooled Positive $L R=12.93(6.28$ to 26.64$)$ $=94.59 ; \mathrm{df}=16(\mathrm{p}=0.0000)$ nconsistency (1-square) $=83.1 \%$
B

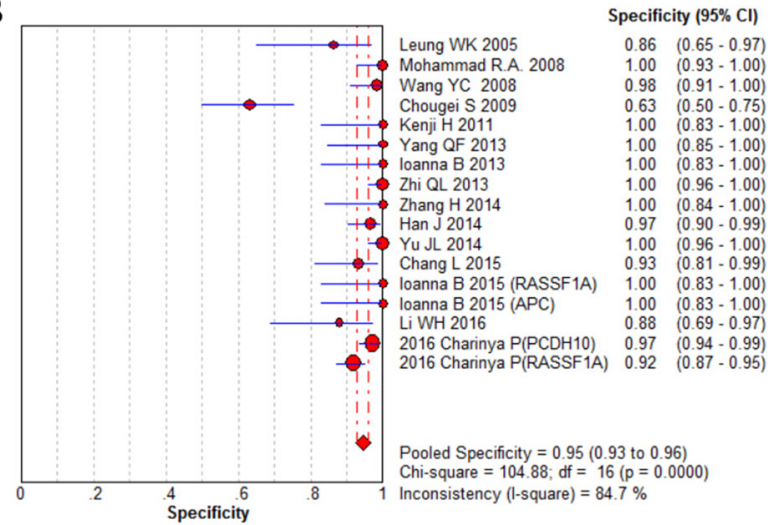

D

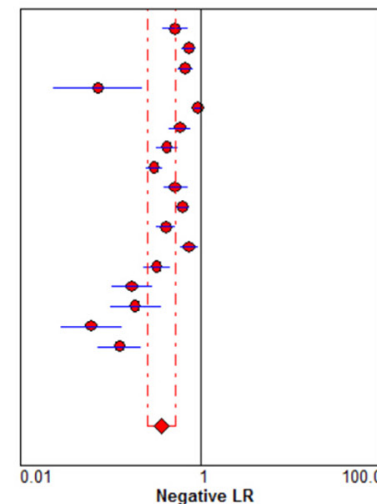

Figure 4: Diagnostic accuracy forest plots. (A) Forest plots of overall sensitivity. (B) Forest plots of overall specificity. (C) Forest plots of positive likelihood ratio. (D) Forest plots of negative likelihood ratio. 
Table 2A: Subgroup analysis of diagnosis measures

\begin{tabular}{|c|c|c|c|c|c|c|c|c|c|}
\hline \multirow[t]{2}{*}{ Subgroup } & \multicolumn{3}{|c|}{ Sensitivity } & \multicolumn{3}{|c|}{ Specificity } & \multicolumn{3}{|c|}{ Diagnostic ratios } \\
\hline & Value & $I^{2}(\%)$ & $\boldsymbol{P}$ & Value & $I^{2}(\%)$ & $\boldsymbol{P}$ & Value & $I^{2}(\%)$ & $\boldsymbol{P}$ \\
\hline \multicolumn{10}{|l|}{ Method } \\
\hline MSP & $0.66(0.63-0.69)$ & 93.3 & 0.000 & $0.96(0.95-0.97)$ & 70.6 & 0.0001 & $58.16(23.44-144.29)$ & 69.3 & 0.0001 \\
\hline qPCR & $0.46(0.39-0.52)$ & 98.4 & 0.000 & $0.85(0.79-0.90)$ & 94.5 & 0.000 & $21.82(9.38-50.76)$ & 0.0 & 0.413 \\
\hline \multicolumn{10}{|l|}{ Race } \\
\hline Mongolian & $0.62(0.59-0.65)$ & 96.0 & 0.000 & $0.94(0.92-0.95)$ & 86.9 & 0.000 & $39.82(17.02-93.15)$ & 72.5 & 0.000 \\
\hline Caucasian & $0.62(0.56-0.68)$ & 93.2 & 0.000 & $1.00(0.97-1.00)$ & 0.0 & 1.000 & $79.01(18.97-328.98)$ & 0.0 & 0.870 \\
\hline \multicolumn{10}{|l|}{ Size } \\
\hline$<65$ & $0.46(0.41-0.52)$ & 86.9 & 0.000 & $0.96(0.93-0.98)$ & 61.4 & 0.017 & $15.43(7.89-30.19)$ & 0.0 & 0.431 \\
\hline$\geq 65$ & $0.67(0.64-0.70)$ & 96.4 & 0.000 & $0.94(0.92-0.96)$ & 89.8 & 0.000 & $80.92(32.86-199.28)$ & 61.3 & 0.006 \\
\hline \multicolumn{10}{|l|}{ Gene target } \\
\hline Single & $0.68(0.58-0.76)$ & 90.2 & 0.001 & $0.87(0.74-0.95)$ & 0.0 & 0.867 & $16.45(3.57-75.79)$ & 59.6 & 0.116 \\
\hline Combined & $0.61(0.59-0.64)$ & 95.8 & 0.000 & $0.95(0.93-0.96)$ & 86.1 & 0.000 & $53.31(24.26-117.13)$ & 61.8 & 0.0008 \\
\hline
\end{tabular}

$r^{2}=$ inconsistency index; MSP = methylation-specific PCR; qMSP = quantitative methylation-specific PCR.

Table 2B: Meta-regression results of diagnostic value

\begin{tabular}{lccccccc}
\hline \multirow{2}{*}{ Parameter } & \multicolumn{3}{c}{ Sensitivity } & & \multicolumn{3}{c}{ Specificity } \\
\cline { 2 - 4 } \cline { 6 - 8 } & Coef & $\mathbf{Z}$ & $\boldsymbol{P}$ & & Coef & $\mathbf{Z}$ & $\boldsymbol{P}$ \\
\hline Method & -0.03 & -0.70 & 0.49 & & 3.65 & -0.21 & 0.83 \\
Race & 0.44 & -0.04 & 0.97 & & 30.10 & 0.00 & 1.00 \\
Size & 1.21 & 0.88 & 0.38 & & 4.42 & 0.31 & 0.76 \\
Gene target & 2.07 & 2.37 & 0.02 & & 2.74 & -1.09 & 0.27 \\
\hline
\end{tabular}

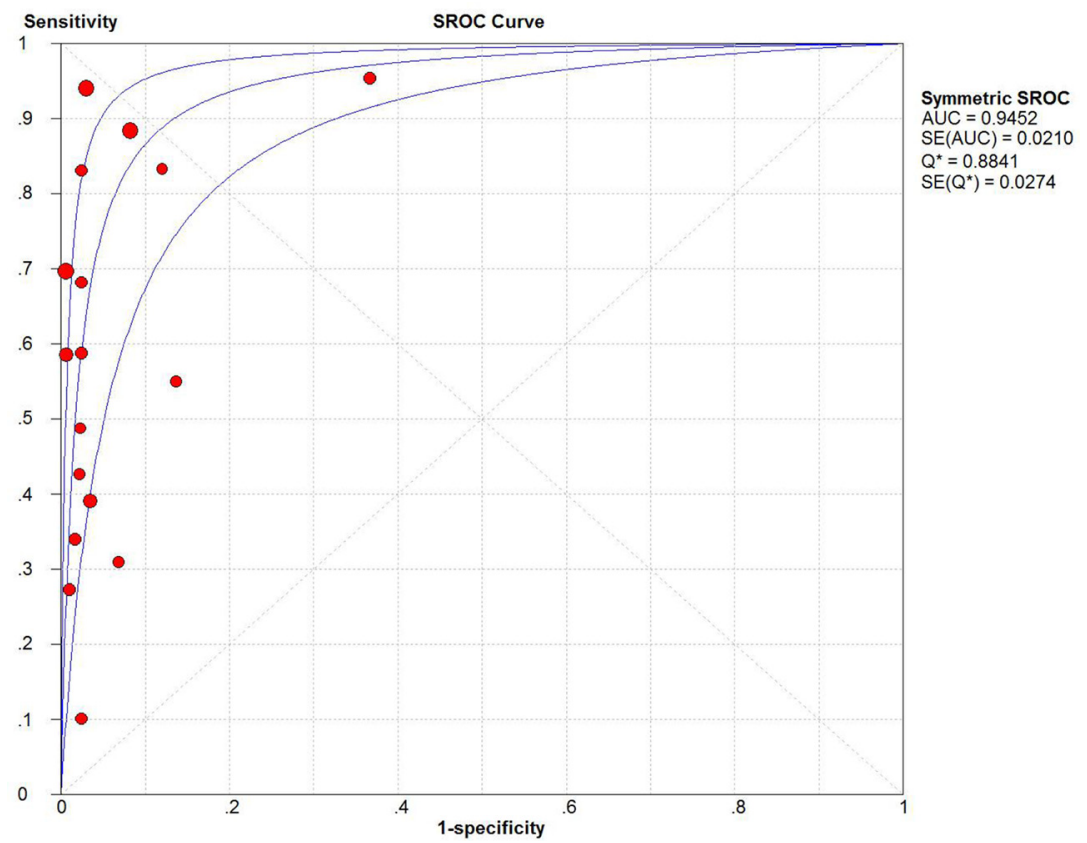

Figure 5: Summary receiver operating characteristic plot for the included studies with the associated $95 \%$ confidence region. 
A

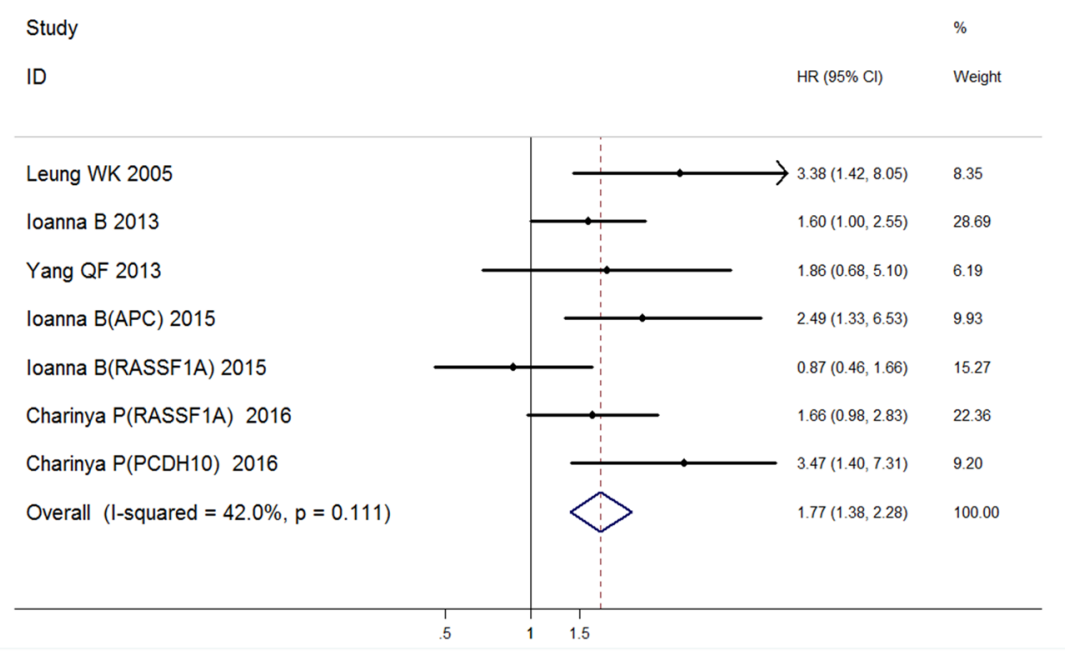

B

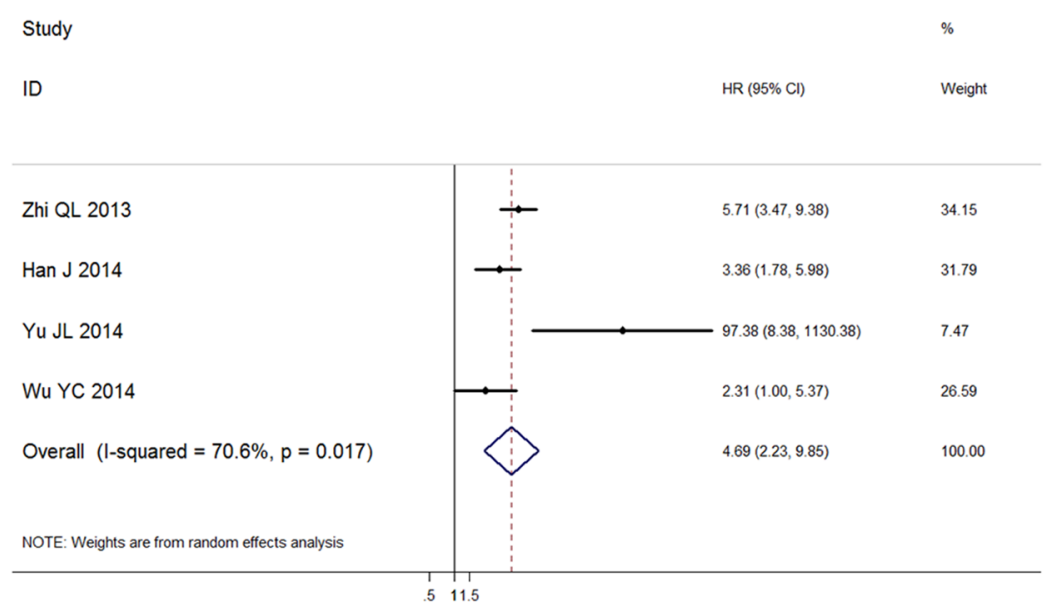

Figure 6: Forest plot of the HRs for survival in ctDNA detection of GC patients. (A) Association with overall survival; (B) Association with disease free survival.

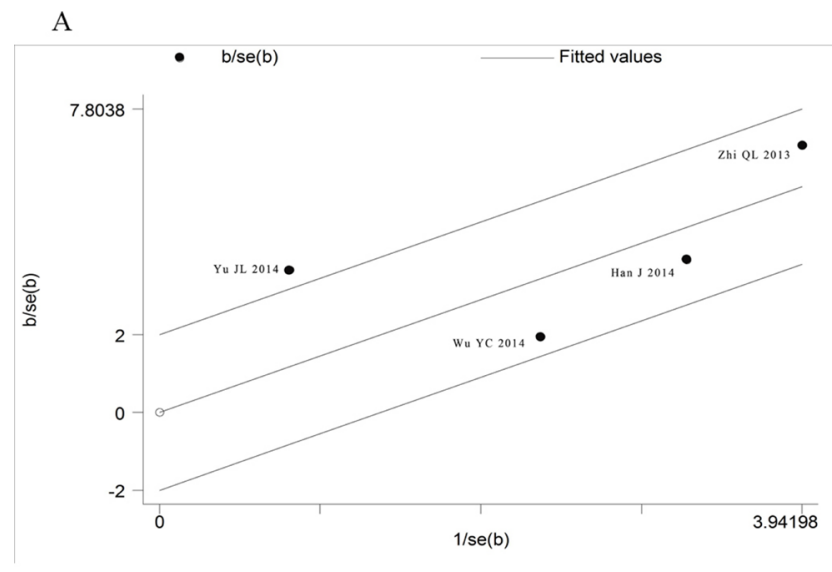

B
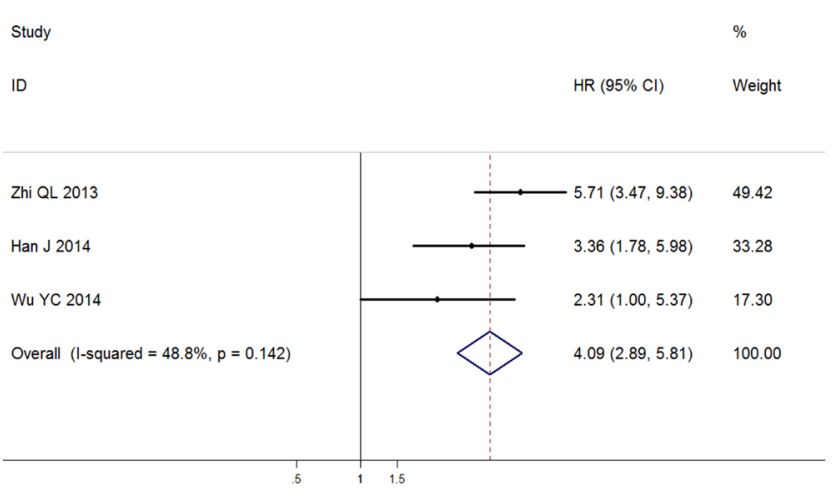

Figure 7: Heterogeneity exploration in DFS analysis. (A) Galbraith blot of association between ctDNA and disease free survival; (B) Forest plot of HRs for disease free survival after omission of Yu JL's study. 
Table 3: Meta-analysis of the association between ctDNA presence and clinicopathological features of GC patients

\begin{tabular}{|c|c|c|c|c|c|c|c|}
\hline \multirow{2}{*}{ Stratification } & \multirow{2}{*}{ No. of studies } & \multirow{2}{*}{ No. of patients } & \multirow{2}{*}{ Pooled OR } & \multirow{2}{*}{$95 \%$ CI of pooled OR } & \multirow{2}{*}{$P$ value } & \multicolumn{2}{|c|}{ Heterogeneity } \\
\hline & & & & & & $I^{2}(\%)$ & $P$-value \\
\hline SEX $(\mathrm{M} / \mathrm{F})$ & 12 & 876 & 0.97 & $0.72-1.31$ & 0.849 & 0 & 0.763 \\
\hline $\mathrm{pT}(\mathrm{I}+\mathrm{II} / \mathrm{III}+\mathrm{IV})$ & 8 & 545 & 0.18 & $0.07-0.45$ & 0.001 & 79.6 & 0.000 \\
\hline Lymph node metastasis (N0/N1-3) & 7 & 744 & 0.19 & $0.06-0.64$ & 0.008 & 90.2 & 0.000 \\
\hline Distant metastasis (M0/M1) & 7 & 606 & 0.32 & $0.20-0.53$ & $0 . .000$ & 41.5 & 0.072 \\
\hline 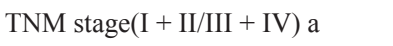 & 6 & 561 & 0.11 & $0.07-0.17$ & 0.000 & 90.4 & 0.000 \\
\hline Tumor size $(<5 \mathrm{~cm} /<5 \mathrm{~cm})$ & 4 & 665 & 0.26 & $0.11-0.61$ & 0.002 & 79.9 & 0.002 \\
\hline $\begin{array}{l}\text { Lauren's classification } \\
\text { (Intestinal/Diffuse) }\end{array}$ & 2 & 317 & 0.89 & $0.57-1.4$ & 0.628 & 0.000 & 0.808 \\
\hline $\begin{array}{l}\text { H. pylori infection } \\
\text { (Negative/positive) }\end{array}$ & 3 & 386 & 0.57 & $0.36-0.91$ & 0.018 & 10.2 & 0.328 \\
\hline
\end{tabular}

a: All the enrolled studies applied AJCC/UICC 7th TNM staging system, except Wang YC's and Chouhei S' studies using the 6th edition. OR: odds ratio; I²: inconsistency index; MSP: methylation-specific PCR; qMSP: quantitative methylation-specific PCR; H. pylori: Helicobacter pylori.

The majority of the gene alteration types in this study were gene methylation/hypermethylation, which might result in inappropriate silencing of tumor suppressor genes. DNA methylation is relatively chemically stable and can be easily detected with a sensitivity of up to $1: 1000$ molecules [33]. In our meta-analysis, the methylation of $A P C, P 16$ or RASSF1A genes were investigated in more than one independent studies. Taken $A P C$ as an example, it was first identified as the cause of the familial adenomatous polyposis syndrome and its dysfunction was closely associated with several gastrointestinal diseases [34]. Methylation-induced dysfunction of $A P C$ and subsequent activation of downstream pathways, such as the Wnt/ $\beta$-catenin pathway, may be responsible for the aggressive tumor behavior [35].Consistent with the gene alteration types, the most common method of methylation detection is MSP or qMSP. The MSP technique is a useful procedure because of its high sensitivity and specificity [36]. Another gene alteration type described above is gene mutation, which could be detected by whole-genome sequence or genotyping technology [37].
Several limitations in this study should be addressed. First, the lack of a well-accepted ctDNA gene target in $\mathrm{GC}$ patients might contribute to the presence of bias. GC is considered as a malignancy with high histological and etiological heterogeneity. Therefore, more circulating genes customized by up-to-date molecular characterization would contribute to ctDNA detection and its clinical application in GC. Owing to the nature of our research, selection bias might occur with enrichment of studies reporting positive results. Furthermore, the difference in detection method and materials, such as PCR primers or the equipment applied, is also an important source of study bias. We also did not have enough information for comparing the ctDNA change before and after surgery, which could restrain the clinical application of ctDNA. Last, the majority of our enrolled studies came from East Asia countries, therefore, our conclusion might not be universal suitable.

Despite its preliminary nature, this study clearly indicated that ctDNA detection might be a specific, but low sensitive test in GC patients. The presence of ctDNA in
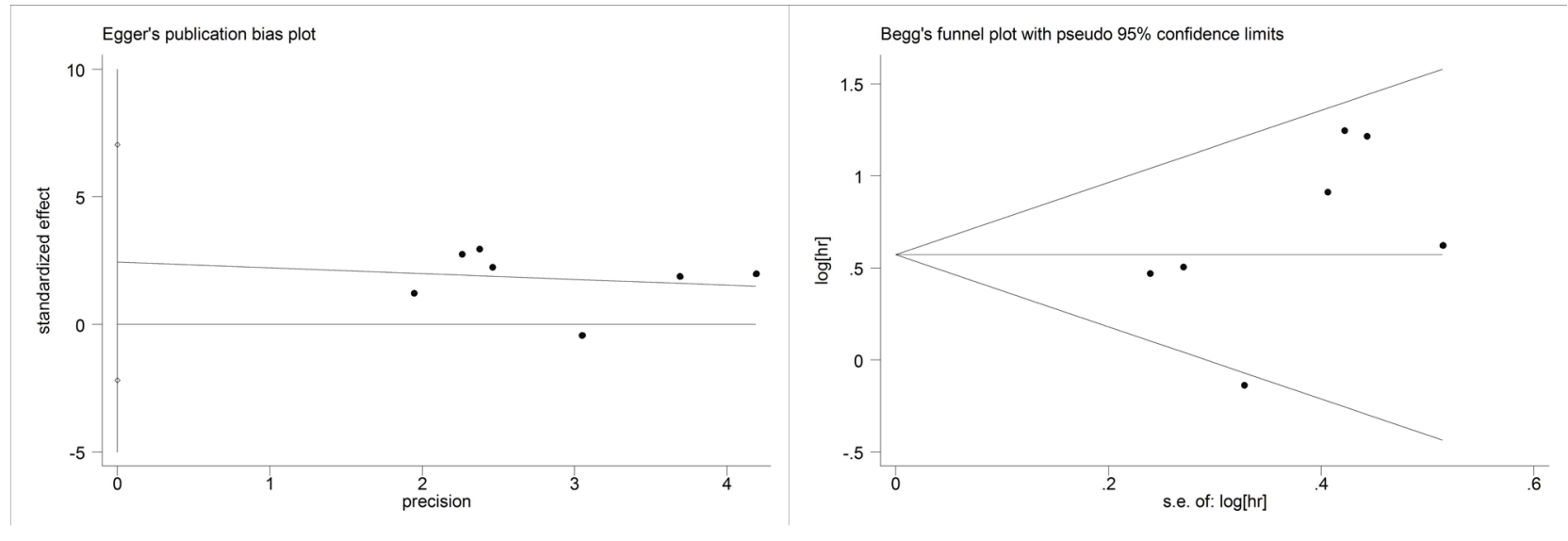

Figure 8: Funnel plot for the evaluation of potential publication bias in the impact of ctDNA on overall survival of GC patients. (A) Begg's funnel plot; (B) Egger's funnel plot. 
GC patients predicted worse TNM stage and unfavorable survival. Before its wide application in GC patients, some concerns still need to be addressed, including more accurate molecule targets, suitable detection techniques. More prospective studies with consistent and standardized methodology are needed to further resolve these problems.

\section{MATERIALS AND METHODS}

\section{Literature search}

The search was conducted by searching the electronic databases Pubmed, Embase, Cochrane Library and Web of Science for all relevant papers published up to November 30, 2016. The following terms were used for searching: "Circulating tumor DNA" or "ctDNA" or "Blood/Serum/plasma DNA" and "gastric/stomach cancer" or "gastric/stomach tumor". Article language was limited to English. Two researchers independently assessed the eligibility of the potential relevant studies by screening the titles and abstracts, and disagreements were solved by discussion. The references of all relevant papers were also checked to retrieve more eligible studies. The Preferred Reporting Items for Systematic Reviews and Meta-analysis (PRISMA) statement [38] was applied as the template for the searching process.

\section{Inclusion criteria}

In this meta-analysis, eligible studies were selected according to the following inclusion criteria: (1) at least one of the diagnostic or prognostic value of ctDNA detection in $\mathrm{GC}$ patients was reported or able to be calculated from published data; (2) samples were collected from the peripheral blood; (3) the techniques and target gene were clearly stated in articles; and (4) studies must include negative controls.

\section{Exclusion criteria}

The exclusion criteria were as follows: (1) studies published in languages other than English; (2) repeated or overlapping publications that included the same population and gene; (3) studies with a poor sample size $(\leq 10)$; and (4) experiments only based on cell lines rather than clinical samples.

\section{Quality assessment}

Two researchers independently reviewed and evaluated all eligible studies according to the NewcastleOttawa scale (NOS) [39]. This scale is an eight-item instrument that made assessment of patient population and selection, study comparability, and outcomes. And the 'baseline characteristics of patients' including patients' gender, age and TNM stage were chosen as the 'important factors' in the Comparability section. We considered a study awarded five or more stars as a high-quality one.

The methodological quality of diagnosis part in this study was assessed by means of the revised Quality Assessment of Diagnostic Accuracy Studies (QUADAS-2) criteria [40]. The criteria consist of four key domains including patient selection, index test, reference standard and flow and timing. All four domains deal with the risk of bias while the first three domains also discuss concerns regarding applicability. Signaling questions are raised to assist measurement of bias, which are answered as "low risk", "high risk" and "unclear risk".

\section{Data extraction}

The following items were extracted from the identified articles: title, name of the first author, publication year, region/country, clinicopathological features (i.e. gender proportion, sample size, TNM stage), detection details (i.e. target gene, genetic alteration type, detection method, time of sampling, sample volume), and accuracy of diagnostic trial (numbers of positive and negative patients for ctDNA detection). If the eligible studies provided survival data, then HR for OS or DFS and their 95\% CIs were collected either directly from the articles or calculated using the methods illustrated by Parmar et al. [41] and Tierney et al [42].

\section{Statistical analysis}

Diagnostic variables, such as sensitivity, specificity, likelihood ratios (i.e. positive likelihood ratios (PLR), negative likelihood ratios (NLR)), diagnostic ratios (DOR) and the summary receiver operating characteristic curve (SROC) were calculated and analyzed using the Meta-Disc software, version 1.4 [43]. The sensitivity was defined as the proportion of patients with ctDNA presence among all patients confirmed as GC. The specificity was defined as the proportion of patients with negative ctDNA detection among all negative control volunteers without GC. The PLR was calculated as sensitivity/ (1-specificity), while NLR was calculated as 1-sensitivity/specificity. Generally, a PLR $>5.0$ and NLR $<0.2$ was considered clinically significant. DOR was calculated as PLR/NLR, which indicated how much greater the chance of having GC is for the patients with ctDNA presence than for the ones without. The pooled HR and the $95 \%$ CIs for OS or DFS were analyzed by the Stata version 12.0 software (StatCorp, College Station, TX, USA). A significant heterogeneity was observed when $P<0.05$ or $I^{2}>50 \%$, and a random-effect model was used. Otherwise, a fixed-effect model was used.

\section{ACKNOWLEDGMENTS}

This article does not contain any studies with human participants performed by any of the authors. All the analyses were based on previous published studies. 


\section{CONFLICTS OF INTEREST}

The authors declare no competing financial interests.

\section{GRANT SUPPORT}

This work was supported by grants from the National Program on Key Basic Research Project of China (No.2014CBA02002), National Key Research and Development Plan (No. 2016YFC0905302).

National Nature Science Foundation of China (No.81272698, 81672319, 81602507) and Beijing Municipal Science and Technology Project (No.D131100005313010).

\section{REFERENCES}

1. Stewart BW. World Cancer Report 2014. International Agency for Research on Cancer (2015).

2. Ferlay J, Steliarova FE, Lortet TJ, Rosso S, Coebergh JW, Comber H, Forman D, Bray F. Cancer incidence and mortality patterns in Europe: estimates for 40 countries in 2012. Eur J Cancer. 2013; 49:1374-1403.

3. Diaz LA, Bardelli A. Liquid biopsies: genotyping circulating tumor DNA. J Clin Oncol. 2014; 32:579-586.

4. Dawson SJ, Rosenfeld N, Caldas C. Circulating tumor DNA to monitor metastatic breast cancer. N Engl J Med. 2013; 369:93-94.

5. Qiu M, Wang J, Xu Y, Ding X, Li M, Jiang F, Xu L, Yin R. Circulating tumor DNA is effective for the detection of EGFR mutation in non-small cell lung cancer: a meta-analysis. Cancer Epidemiol Biomarkers Prev. 2015; 24:206-212.

6. Bettegowda C, Sausen M, Leary RJ, Kinde I, Wang Y, Agrawal N, Bartlett BR, Wang H, Luber B, Alani RM, Antonarakis ES, Azad NS, Bardelli A, et al. Detection of circulating tumor DNA in early- and late-stage human malignancies. Sci Trans1 Med. 2014; 6:224ra24.

7. Cheng F, Su L, Qian C. Circulating tumor DNA: a promising biomarker in the liquid biopsy of cancer. Oncotarget; Oncotarget. 2016; 7:48832-48841. doi: 10.18632/ oncotarget.9453.

8. Leung WK, To KF, Chu ES, Chan MW, Bai AH, Ng EK, Chan FK, Sung JJ. Potential diagnostic and prognostic values of detecting promoter hypermethylation in the serum of patients with gastric cancer. Br J Cancer. 2005; 92:2190-2194.

9. Abbaszadegan MR, Moaven O, Sima HR. P16 promoter hypermethylation: a useful serum marker for early detection of gastric cancer. World J Gastroenterol. 2008; 14:2055-2060.

10. Wang YC, Yu ZH, Liu C, Xu LZ, Yu W, Lu J, Zhu RM, Li GL, Xia XY, Wei XW, Ji HZ, Lu H, Gao Y, et al. Detection of RASSF1A promoter hypermethylation in serum from gastric and colorectal adenocarcinoma patients. World J Gastroenterol. 2008; 14:3074-3080.

11. Sakakura C, Hamada $T$, Miyagawa $K$, Nishio $M$, Miyashita A, Nagata H, Ida H, Yazumi S, Otsuji E,
Chiba T, Ito K, Ito Y. Quantitative analysis of tumor-derived methylated RUNX3 sequences in the serum of gastric cancer patients. Anticancer Res. 2009; 29:2619-2625.

12. Hibi K, Goto $T$, Shirahata A, Saito M, Kigawa G, Nemoto H, Sanada Y. Detection of TFPI2 methylation in the serum of gastric cancer patients. Anticancer Res. 2011; $31: 3835-3838$.

13. Balgkouranidou I, Karayiannakis A, Matthaios D, Bolanaki H, Tripsianis G, Tentes AA, Lianidou E, Chatzaki E, Fiska A, Lambropoulou M, Kolios G, Kakolyris S. Assessment of SOX17 DNA methylation in cell free DNA from patients with operable gastric cancer. Association with prognostic variables and survival. Clin Chem Lab Med. 2013; 51:1505-1510.

14. Yang Q, Gao J, Xu L, Zeng Z, Sung JJ, Yu J. Promoter hypermethylation of BCL6B gene is a potential plasma DNA biomarker for gastric cancer. Biomarkers. 2013; 18:721-725.

15. Ling Z.Q. Lv P, Lu XX, Yu JL, Han J, Ying LS, Zhu X, Zhu WY, Fang XH, Wang $\mathrm{S}$, Wu YC. Circulating Methylated XAF1 DNA Indicates Poor Prognosis for Gastric Cancer. PLoS One. 2013; 8:e67195.

16. Han J, Lv P, Yu JL, Wu YC, Zhu X, Hong LL, Zhu WY, Yu QM, Wang XB, Li P, Ling ZQ. Circulating methylated MINT2 promoter DNA is a potential poor prognostic factor in gastric cancer. Dig Dis Sci. 2014; 59:1160-1168.

17. Wu Y C, Lv P, Han J, Yu JL, Zhu X, Hong LL, Zhu WY, Yu QM, Wang XB, Li P, Ling ZQ. Enhanced serum methylated p16 DNAs is associated with the progression of gastric cancer. International Journal of Clinical \& Experimental Pathology. 2014; 7:1553-1562.

18. Yu JL, Lv P, Han J, Zhu X, Hong LL, Zhu WY, Wang XB, Wu YC, Li P, Ling ZQ. Methylated TIMP-3 DNA in body fluids is an independent prognostic factor for gastric cancer. Arch Pathol Lab Med. 2014; 138:1466-1473.

19. Zhang H, Song Y, Xia P, Cheng Y, Guo Q, Diao D, Wang W, Wu X, Liu D, Dang C. Detection of aberrant hypermethylated spastic paraplegia-20 as a potential biomarker and prognostic factor in gastric cancer. Med Oncol. 2014; 31:830.

20. Liu C, Li N, Lu H, Wang Z, Chen C, Wu L, Liu J, Lu Y, Wang F. Circulating SFRP1 promoter methylation status in gastric adenocarcinoma and esophageal square cell carcinoma. Biomed Rep. 2015; 3:123-127.

21. Balgkouranidou I, Matthaios D, Karayiannakis A, Bolanaki H, Michailidis P, Xenidis N, Amarantidis K, Chelis L, Trypsianis G, Chatzaki E, Lianidou ES, Kakolyris S. Prognostic role of APC and RASSF1A promoter methylation status in cell free circulating DNA of operable gastric cancer patients. Mutat Res. 2015; 778:46-51.

22. Pimson C, Ekalaksananan T, Pientong C, Promthet S, Putthanachote N, Suwanrungruang K, Wiangnon S. Aberrant methylation of PCDH10andRASSF1Agenes in blood samples for non-invasive diagnosis and prognostic assessment of gastric cancer. PeerJ. 2016; 4:e2112. 
23. Li WH, Zhou ZJ, Huang TH, Guo K, Chen W, Wang Y, Zhang H, Song YC, Chang DM. Detection of OSR2, VAV3, and PPFIA3 Methylation in the Serum of Patients with Gastric Cancer. Dis Markers. 2016; 5780538.

24. Mocellin S, Verdi D, Pooley KA, Nitti D. Genetic variation and gastric cancer risk: a field synopsis and meta-analysis. Gut. 2015; 64:1209-1219.

25. Diehl F, Schmidt K, Choti MA, Romans K, Goodman S, Li M, Thornton K, Agrawal N, Sokoll L, Szabo SA, Kinzler KW, Vogelstein B, Diaz LJ. Circulating mutant DNA to assess tumor dynamics. Nat Med. 2008; 14:985-990.

26. Tsujiura M, Ichikawa D, Konishi H, Komatsu S, Shiozaki A, Otsuji E. Liquid biopsy of gastric cancer patients: circulating tumor cells and cell-free nucleic acids. World J Gastroenterol. 2014; 20:3265-3286.

27. Mandel P, Metais P. Les acides nucléiques du plasma sanguin chez l'homme. C R Seances Soc Biol Fil. 1948; 142:241-243.

28. Engelman JA, Mukohara T, Zejnullahu K, Lifshits E, Borrás AM, Gale CM, Naumov GN, Yeap BY, Jarrell E, Sun J, Tracy S, Zhao X, Heymach JV, et al. Allelic dilution obscures detection of a biologically significant resistance mutation in EGFR-amplified lung cancer. J Clin Invest. 2006; 116:2695-2706.

29. Yang AP, Liu J, Lei HY, Zhang QW, Zhao L, Yang GH. CA72-4 combined with CEA, CA125 and CA19-9 improves the sensitivity for the early diagnosis of gastric cancer. Clin Chim Acta. 2014; 437:183-186.

30. Swets JA. Measuring the accuracy of diagnostic systems. Science. 1988; 240:1285-1293.

31. Zhou Q, Zheng X, Chen L, Xu B, Yang X, Jiang J, Wu C. Smad2/3/4 Pathway Contributes to TGF- $\beta$-Induced MiRNA-181b Expression to Promote Gastric Cancer Metastasis by Targeting Timp3. Cell Physiol Biochem. 2016; 39:453-466.

32. Engelman JA. Targeting PI3K signalling in cancer: opportunities, challenges and limitations. Nat Rev Cancer. 2009; 9:550-562.

33. Ye T, Chen Y, Fang J. DNA methylation biomarkers in serum for gastric cancer screening. Mini Rev Med Chem. 2010; 10:1034-1038.
34. Tamura G, Sato K, Akiyama S, Tsuchiya T, Endoh Y, Usuba O, Kimura W, Nishizuka S, Motoyama T. Molecular characterization of undifferentiated-type gastric carcinoma. Lab Invest. 2001; 81:593-598.

35. Janssen KP, Alberici P, Fsihi H, Gaspar C, Breukel C, Franken P, Rosty C, Abal M, El Marjou F, Smits R, Louvard D, Fodde R, Robine S. APC and oncogenic KRAS are synergistic in enhancing Wnt signaling in intestinal tumor formation and progression. Gastroenterology. 2006; 131:1096-109.

36. Herman JG, Graff JR, Myöhänen S, Nelkin BD, Baylin SB. Methylation-specific PCR: a novel PCR assay for methylation status of $\mathrm{CpG}$ islands. Proc Natl Acad Sci USA. 1996; 93:9821-9826.

37. Nakagawa H, Wardell CP, Furuta M, Taniguchi $H$, Fujimoto A. Cancer whole-genome sequencing: present and future. Oncogene. 2015; 34:5943-5950.

38. Moher D, Liberati A, Tetzlaff J, Altman DG, Group P. Preferred reporting items for systematic reviews and metaanalyses:the PRISMA statement. Ann Intern Med. 2009; 151:264-269.

39. Stang A. Critical evaluation of the Newcastle-Ottawa scale for the assessment of the quality of nonrandomized studies in meta-analyses. Eur J Epidemiol. 2010; 25:603-605.

40. Whiting PF, Rutjes AW, Westwood ME, Mallett S, Deeks JJ, Reitsma JB, Leeflang MM, Sterne JA, Bossuyt PM, QUADAS-2 Group. QUADAS-2: a revisedtool for the quality assessment of diagnostic accuracy studies. Annals of internal medicine. 2011; 155:529-536.

41. Parmar MK, Torri V, Stewart L. Extracting summary statistics to perform meta-analyses of the published literature for survival endpoints. Stat Med. 1998:17:2815-2834.

42. Tierney JF, Stewart LA, Ghersi D, Burdett S, Sydes MR. Practical methods for incorporating summary time-to-event data into meta-analysis. Trials. 2007; 8:16.

43. Zamora J, Abraira V, Muriel A, Khan K, Coomarasamy A. Meta-DiSc: a software for meta-analysis of test accuracy data. BMC Med Res Methodol. 2006; 6:31. 